\title{
Combined Oral Contraceptives in Women with Systemic Lupus Erythematosus
}

\author{
Michelle Petri, M.D., M.P.H., Mimi Y. Kim, Sc.D., Kenneth C. Kalunian, M.D., \\ Jennifer Grossman, M.D., Bevra H. Hahn, M.D., Lisa R. Sammaritano, M.D., \\ Michael Lockshin, M.D., Joan T. Merrill, M.D., H. Michael Belmont, M.D., \\ Anca D. Askanase, M.D., M.P.H., W. Joseph McCune, M.D., \\ Michelene Hearth-Holmes, M.D., Mary Anne Dooley, M.D., M.P.H., \\ Joan Von Feldt, M.D., Alan Friedman, M.D., Mark Tan, M.D., \\ John Davis, M.D., M.P.H., Mary Cronin, M.D., Betty Diamond, M.D., \\ Meggan Mackay, M.D., Lisa Sigler, M.A., Michael Fillius, B.S., Ann Rupel, B.A., \\ Frederick Licciardi, M.D., and Jill P. Buyon, M.D., for the OC-SELENA Trial*
}

\section{ABSTRACT}

From Johns Hopkins University Schoo of Medicine, Baltimore (M.P., L.S. M.F.); Albert Einstein College of Medicine-Montefiore Medical Center, Bronx, N.Y. (M.Y.K., B.D., M.M.); University of California, Los Angeles (K.C.K., J.G., B.H.H.); Hospital for Special Surgery, New York (L.R.S., M.L.); Oklahoma Medical Research Foundation, Oklahoma City (J.T.M.); the Hospital for Joint Diseases and Bellevue Hospital at New York University School of Medicine, New York (H.M.B., A.D.A., A.R., F.L., J.P.B.); University of Michigan, Ann Arbor (W.J.M.); Louisiana State University Health Sciences Center, Shreveport (M.H.-H.); University of North Carolina at Chapel Hill, Chape Hill (M.A.D.); University of Pennsylvania Philadelphia (J.V.F.); University of Texas, Houston (A.F.); Rheumatology Associates of Long Island, Port Jefferson Station N.Y. (M.T.); University of California, San Francisco, San Francisco (J.D.); and Medical College of Wisconsin, Milwaukee (M.C.). Address reprint requests to $\mathrm{Dr}$ Buyon at the Department of Rheumatology, Rm. 1608, Hospital for Joint Diseases, 301 E. 17th St., New York, NY 10003, or at jill.buyon@nyumc.org.

*Additional investigators who participated in the Oral Contraceptives-SELENA (OCSELENA) Trial are listed in the Appendix.

N Engl J Med 2005;353:2550-8.

Copyright @ 2005 Massachusetts Medical Society.

\section{BACKGROUND}

Oral contraceptives are rarely prescribed for women with systemic lupus erythematosus, because of concern about potential negative side effects. In this double-blind, randomized, noninferiority trial, we prospectively evaluated the effect of oral contraceptives on lupus activity in premenopausal women with systemic lupus erythematosus.

\section{METHODS}

A total of 183 women with inactive (76 percent) or stable active (24 percent) systemic lupus erythematosus at 15 U.S. sites were randomly assigned to receive either oral contraceptives (triphasic ethinyl estradiol at a dose of $35 \mu \mathrm{g}$ plus norethindrone at a dose of 0.5 to $1 \mathrm{mg}$ for 12 cycles of 28 days each; 91 women) or placebo ( 92 women) and were evaluated at months 1, 2, 3, 6, 9, and 12. Subjects were excluded if they had moderate or high levels of anticardiolipin antibodies, lupus anticoagulant, or a history of thrombosis.

\section{RESULTS}

The primary end point, a severe lupus flare, occurred in 7 of 91 subjects receiving oral contraceptives ( 7.7 percent) as compared with 7 of 92 subjects receiving placebo ( 7.6 percent). The 12-month rates of severe flare were similar: 0.084 for the group receiving oral contraceptives and 0.087 for the placebo group ( $\mathrm{P}=0.95$; upper limit of the one-sided 95 percent confidence interval for this difference, 0.069 , which is within the prespecified 9 percent margin for noninferiority). Rates of mild or moderate flares were 1.40 flares per person-year for subjects receiving oral contraceptives and 1.44 flares per person-year for subjects receiving placebo (relative risk, $0.98 ; \mathrm{P}=0.86$ ). In the group that was randomized to receive oral contraceptives, there was one deep venous thrombosis and one clotted graft; in the placebo group, there was one deep venous thrombosis, one ocular thrombosis, one superficial thrombophlebitis, and one death (after cessation of the trial).

\section{CONCLUSIONS}

Our study indicates that oral contraceptives do not increase the risk of flare among women with systemic lupus erythematosus whose disease is stable. 
ONCERN REGARDING POTENTIAL NEGative effects of exogenous estrogens on the course of systemic lupus erythematosus has influenced prescribing practices, although placebocontrolled trials have not been performed. The basis of reluctance to prescribe oral contraceptives is that the incidence of systemic lupus erythematosus in women is 10 times that in men, ${ }^{1}$ that the disease generally presents after menarche and before menopause, and that estrone metabolism in lupus is skewed toward 16 $\alpha$-hydroxylated compounds that are themselves potent estrogens. ${ }^{2}$ Lupus flares have occurred in patients receiving exogenous hormones, ${ }^{3}$ and a retrospective study among subjects with preexisting renal disease suggested adverse effects. ${ }^{4}$ The administration of estrogen exacerbates lupus in mice ${ }^{5}$ and, depending on the genetic background, augments the survival and autoreactivity of murine B cells. ${ }^{6}$

Despite the preceding data, there are settings in which exogenous estrogens might provide benefit. Premenopausal women with systemic lupus erythematosus should have access to safe and effective birth control. Women who have diminished fertility may seek hormonal manipulation to stimulate ovulation, and women receiving cyclophosphamide may need methods for preserving fertility. Furthermore, exogenous estrogens may be used to prevent glucocorticoid-induced osteoporosis and to treat ovarian cysts, endometriosis, irregular menses, and menometrorrhagia. The high rate of elective abortion among women with systemic lupus erythematosus (about 23 percent of pregnancies) may reflect the failure of the birth-control method used or the absence of an adequate birth-control program. ${ }^{7}$

To address these issues, we initiated the Safety of Estrogens in Lupus Erythematosus National Assessment (SELENA) trial, which comprised two separate, randomized, placebo-controlled, multicenter studies (one of hormone replacement ${ }^{8}$ and the other of combined oral contraceptives [OC-SELENA]). The goal of the OC-SELENA trial was to determine the effect of oral contraceptives containing low-dose synthetic estrogens and progestins on disease activity in premenopausal women with systemic lupus erythematosus. The study was designed as a noninferiority trial to establish that oral contraceptives do not appreciably increase the risk of a severe flare as compared with placebo.

\section{METHODS}

\section{STUDY DESIGN}

Investigators from the core SELENA group 3 met before the recruitment of patients to revise the definitions of the instrument used, the Systemic Lupus Erythematosus Disease Activity Index (SLEDAI). ${ }^{9}$ The SELENA-SLEDAI flare composite 8,10 included three elements: the SELENA-SLEDAI score (range, 0 to 105 , with 0 indicating inactive disease); an assessment of new or worsening disease activity, medication changes, and hospitalizations not captured with the use of the SLEDAI; and the score on the physician's global-assessment visual-analogue scale (range, 0 to 3 , with 0 indicating inactive disease and 3 severe disease) (for details, see the Supplementary Appendix, available with the full text of this article at www.nejm.org). The physicians at each study site scored each element of the flare composite. Mild or moderate flares and severe flares were defined exactly as described in the recently published hormone-replacement study of the SELENA trial. ${ }^{8}$ Whether these definitions could identify flares accurately and uniformly was tested before enrollment with the use of scenarios involving subjects from the Hopkins Lupus Cohort. ${ }^{11}$ In 2001, all site investigators completed this test with the use of case-report forms of SELENA subjects, which showed a high intraclass correlation (0.89).

\section{PATIENT POPULATION}

A total of 183 women with systemic lupus erythematosus were enrolled from June 1997 through July 2002 (with follow-up through July 2003) at 15 U.S. sites (see the Supplementary Appendix). Institutional review boards at all sites approved the protocol and consent forms, and written informed consent was obtained from subjects before enrollment. Throughout the study, each site reported adverse events to its local institutional review board. In addition, all serious adverse events in the overall study were reported to all institutional review boards.

At enrollment, subjects fulfilled at least four of the criteria of the American College of Rheumatology for the classification of systemic lupus erythematosus $^{12}$ and were under 40 years of age if they were nonsmokers or under 36 years of age if they were smokers. All subjects had disease that was 
clinically stable or had improved in the previous three months. Subjects were stratified as having either inactive disease (defined as a SELENA-SLEDAI score of 4 or less and the receipt of a daily dose of prednisone of no more than $0.5 \mathrm{mg}$ per kilogram of body weight that had not been increased in the previous three weeks) or stable active disease (defined as a SELENA-SLEDAI score of 5 to 12 and the receipt of a daily dose of prednisone of no more than $0.5 \mathrm{mg}$ per kilogram that had not been increased in the previous three weeks). Additional immunosuppressive drugs at enrollment were permitted if the dose had been stable for the previous two months. Exclusion criteria were the use of oral contraceptives for more than one month after the diagnosis of systemic lupus erythematosus; a diastolic blood pressure of more than $95 \mathrm{~mm} \mathrm{Hg}$ or a systolic blood pressure of more than $145 \mathrm{~mm} \mathrm{Hg}$ on three determinations; a history of spontaneous deep venous thrombosis, arterial thrombosis, or pulmonary embolus; the presence of IgG, IgM, or IgA anticardiolipin antibodies (more than 40 IgG phospholipid units, 40 IgM phospholipid units, or 50 IgA phospholipid units), a demonstration of lupus anticoagulant by the dilute Russell's vipervenom time test, or both; a history of gynecologic or breast cancer; a history of myocardial infarction; hepatic dysfunction or tumors of the liver; uncontrolled diabetes; congenital hyperlipidemia; migraines associated with neurologic sequelae; unexplained vaginal bleeding; or a positive pregnancy test.

Potential subjects were informed orally by the recruiting physician and in the written consent form that they might receive either oral contraceptives or identical placebo pills. To enter the study, all subjects had to agree to use an alternative form of birth control throughout the study, and any subject who became pregnant was instructed to discontinue the study drug.

\section{RANDOMIZATION AND TREATMENT}

Subjects were randomly assigned in a ratio of $1: 1$ to receive oral contraceptives (triphasic ethinyl estradiol plus norethindrone) (Ortho-Novum 7/7/7, Ortho-McNeil) in four-week cycles (seven days of $35 \mu \mathrm{g}$ of ethinyl estradiol plus $0.5 \mathrm{mg}$ of norethindrone, seven days of $35 \mu \mathrm{g}$ of ethinyl estradiol plus $0.75 \mathrm{mg}$ of norethindrone, seven days of $35 \mu \mathrm{g}$ of ethinyl estradiol plus $1.0 \mathrm{mg}$ of norethindrone, and seven days of inert pills) or identical placebo (OrthoMcNeil). The randomization scheme was stratified according to study site and disease severity (stable active vs. inactive). Permuted blocks of variable size ( 2 through 8 ) were used within each stratum for treatment assignments. Study drug was taken for 12 cycles of 28 days each ("months"). The study drug (Ortho-Novum 7/7/7) and matching placebo were provided free of charge by the manufacturer, Ortho-McNeil; the company had no role in the initiation, planning, conduct, data assembly, analysis, or interpretation of the study.

At screening, a history was taken and physical examination performed. A gynecologic examination and Papanicolaou smear were required within the year preceding enrollment. Laboratory tests included complete blood count, metabolic panel, lipid profile, urinalysis, and 24-hour urine collection to measure levels of creatinine and protein excretion. Serologic profiles included antinuclear antibodies assayed by HEp-2 cell line, anti-double-stranded DNA (anti-dsDNA) antibodies by enzyme-linked immunosorbent assay (ELISA, Diamedix), and C3 and C4 by nephelometry (Dade Behring) at the Hospital for Joint Diseases in New York; lupus anticoagulant by dilute Russell's viper-venom time test with confirmatory mixing studies at Johns Hopkins University in Baltimore; and anticardiolipin antibodies by ELISA (standard $\beta_{2}$-glycoprotein I-dependent assay) at the Hospital for Special Surgery in New York. Subjects were seen at screening and qualifying visits, contacted 2 weeks after entry, and then seen at 1, 2, 3, 6, 9, and 12 months.

\section{END POINTS}

The primary end point was the occurrence of a severe flare. Secondary end points were the occurrence of mild or moderate flares and score on the SELENA-SLEDAI instrument. Pregnancy was not considered as a statistical end point or outcome measure but, rather, constituted a discontinuation criterion. In this noninferiority trial, our design criteria were to demonstrate that oral contraceptives did not increase the risk of the occurrence of a severe flare by more than a prespecified maximum margin that was clinically acceptable, as compared with placebo. We expected a 12-month severe-flare rate of 6 percent in the placebo group. Taking into consideration the potential benefits of oral contraceptives, the SELENA investigators deemed a priori that an absolute difference of less than 9 percent in severe-flare rates between the groups receiving oral contraceptives and placebo would be clinically acceptable. 


\section{STATISTICAL ANALYSIS}

The criterion for the establishment of the safety of oral contraceptives was that the upper limit of the one-sided 95 percent confidence interval for the difference in severe-flare rates between the groups had to be less than 9 percent. It was determined that a sample size of 350 subjects would yield 95 percent power at a one-sided type I error rate of 0.05 to conclude that oral contraceptives are not inferior to placebo, assuming a noninferiority margin of 9 percent and a 6 percent severe-flare rate in both treatment groups. However, the SELENA data and safety monitoring board elected to close enrollment after 183 patients had been randomly assigned, because of difficulty in recruitment.

Distributions of time to the first occurrence of a severe flare were estimated with the use of the Kaplan-Meier method. The difference in 12-month severe-flare rates between treatment groups was computed from the difference in the corresponding Kaplan-Meier estimates.

The Cox proportional-hazards model was used to obtain estimates of the relative risk of severe flare. Times to first occurrences of mild or moderate flares and flares of any type were analyzed with similar approaches. The SELENA-SLEDAI instrument score was analyzed by computing the change from baseline at each follow-up visit and comparing the size of the changes between treatment groups with the use of the two-sample t-test. Linear mixed-effects models were also fitted to the repeated measures.

Analyses were based on the intention-to-treat principle. Data on subjects who were lost to followup were considered to be censored at the time of the subjects' last visit. A per-protocol analysis that was limited to subjects who were fully adherent to the treatment protocol was also performed to evaluate the primary end point. P values are two-sided and are based on the standard null hypothesis of no treatment difference.

\section{RESULTS}

\section{ENROLLMENT}

A total of 91 subjects were randomly assigned to receive oral contraceptives, and 92 subjects to receive placebo. Figure 1 is a flow chart of the study subjects; Table 1 summarizes the subjects' clinical characteristics at enrollment. Racial and ethnic backgrounds, which were determined by the subjects, were similar for subjects in the oral-contraceptive group and the placebo group, respectively: white,

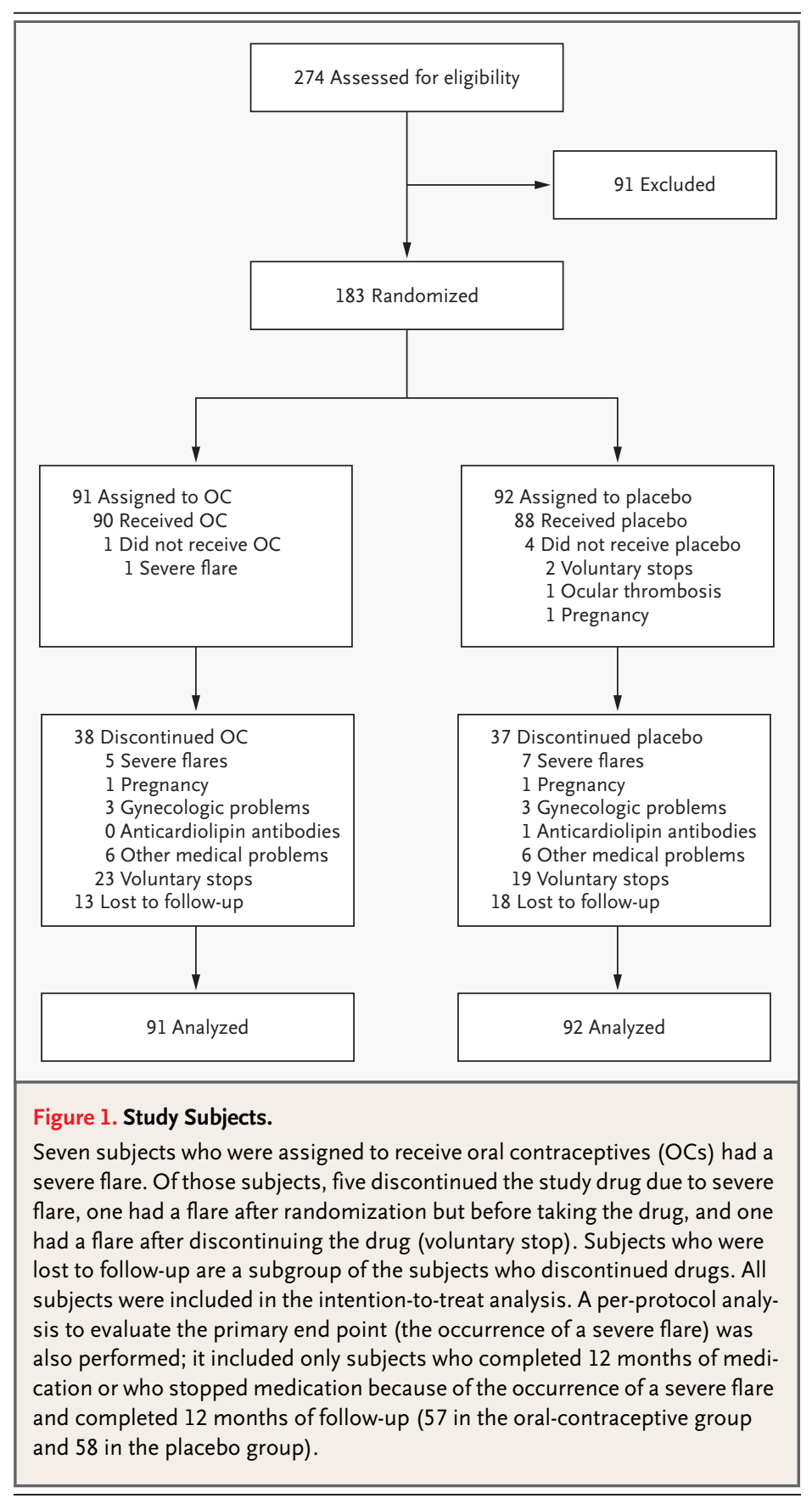

41 percent and 33 percent; black, 28 percent and 38 percent; Asian, 13 percent and 14 percent; Hispanic, 18 percent and 14 percent; and other groups, 1 percent and 1 percent.

The 12-month nonadherence rate was 37.4 percent for the oral-contraceptive group and 37.0 percent for the placebo group $(\mathrm{P}=0.95)$. Subjects who terminated the study drug early for any reason other than the occurrence of a severe flare were 


\begin{tabular}{|c|c|c|}
\hline $\begin{array}{l}\text { Table 1. Baseline Characteristic } \\
\text { According to Treatment Group }\end{array}$ & cs of the Study Su & ibjects \\
\hline Characteristic & $\begin{array}{c}\text { Oral } \\
\text { Contraceptives } \\
(\mathrm{N}=91)\end{array}$ & $\begin{array}{l}\text { Placebo } \\
(\mathrm{N}=92)\end{array}$ \\
\hline \multicolumn{3}{|l|}{ Age $(y r)$} \\
\hline Mean & 29.8 & 30.1 \\
\hline Range & $18-39$ & $18-39$ \\
\hline Active disease (\%) & 23 & 25 \\
\hline \multicolumn{3}{|l|}{$\begin{array}{l}\text { SELENA-SLEDAI Instrument } \\
\text { score }\end{array}$} \\
\hline Mean & 3.01 & 3.39 \\
\hline Range & $0-10$ & $0-12$ \\
\hline Renal disorder (\%) & 37 & 37 \\
\hline Low levels of complement (\%) & 29 & 25 \\
\hline Increased DNA binding (\%) & 32 & 22 \\
\hline Prednisone use (\%) & 56 & 55 \\
\hline \multicolumn{3}{|l|}{ Prednisone dose (mg/day) } \\
\hline Mean & 5.16 & 5.38 \\
\hline Range & $0-30$ & $0-30 \dagger$ \\
\hline
\end{tabular}

* SELENA denotes Safety of Estrogen in Lupus Erythematosus National Assessment, and SLEDAI Systemic Lupus Erythematosus Disease Activity Index.

$\dagger$ One patient was receiving $60 \mathrm{mg}$ of prednisone.

considered to be nonadherent. The 12-month rate of loss to follow-up (i.e., nonadherent subjects who dropped out completely and were not followed for the full 12 months) was 14 percent for the oral-contraceptive group and 20 percent for the placebo group $(\mathrm{P}=0.34)$.

\section{OUTCOMES}

The occurrence of a severe flare was infrequent in both groups: 7 of 91 subjects in the oral-contraceptive group ( 7.7 percent) and 7 of 92 subjects in the placebo group (7.6 percent) (Table 2). Two of the severe flares in the oral-contraceptive group took place when subjects were not taking the study drug (one subject had a flare after qualifying for the study but before taking the drug, and the other had a flare four months after voluntarily stopping the drug). The 12-month severe-flare rate estimated from the Kaplan-Meier approach was 0.084 for the oral-contraceptive group (95 percent confidence interval, 0.024 to 0.14 ) and 0.087 for the placebo group (95 percent confidence interval, 0.025 to 0.15 ), which was a difference of -0.0028 between the groups $(\mathrm{P}=0.95)$. The upper limit of the onesided 95 percent confidence interval for the true difference was 0.069 , implying that the data are consistent with an absolute difference in severeflare rates of up to 6.9 percent, less than the prespecified equivalence margin of 9 percent. A KaplanMeier estimate for the cumulative probability of the occurrence of severe flare is provided in Figure 2. As calculated by the Cox proportional-hazards model, the estimated relative risk of the occurrence of severe flare while receiving oral contraceptives, as compared with placebo, was $0.93(\mathrm{P}=0.89$; 95 percent confidence interval, 0.33 to 2.65). Although the sample size was reduced to 183 subjects, we were able to demonstrate that the difference in the risk of severe flare between the oral-contraceptive group and the placebo group was less than the maximum clinically acceptable difference. Furthermore, this finding persisted even with the hypothetical calculation of one or two additional severe flares in the oral-contraceptive group (data not shown).

The per-protocol analysis, which included 57 subjects receiving oral contraceptives and 58 receiving placebo, yielded an estimated 12-month rate of severe flares of 0.088 for oral contraceptives (95 percent confidence interval, 0.014 to 0.16 ) and 0.12 for placebo (95 percent confidence interval, 0.037 to 0.20$)$. The difference in the 12 -month rate of severe flares between groups was $-0.033(\mathrm{P}=0.56)$. The upper limit of the one-sided 95 percent confidence interval for the true difference was 0.060 , less than the prespecified margin of 9 percent and therefore consistent with the intention-to-treat result that oral contraceptives are not inferior to placebo with respect to risk of a severe flare.

In both study groups, subjects who entered the trial with stable active disease were at increased risk for severe flare as compared with those with inactive disease. The estimated relative risk, adjusted for treatment, was 3.50 (95 percent confidence interval, 1.23 to $9.99 ; \mathrm{P}=0.02$ ).

Rates of mild or moderate flares did not differ significantly between groups: 63 subjects randomized to receive oral contraceptives ( 69 percent) and 55 subjects randomized to receive placebo (60 percent) had one or more mild or moderate flares. The incidence rate for mild or moderate flares, which included multiple flares in the same subject, was 1.40 flares per person-year for the oral-contraceptive group and 1.44 flares per person-year for the placebo group (relative risk, 0.98 ; 95 percent confidence interval, 0.76 to $1.26 ; \mathrm{P}=0.86$ ).

The probability of having at least one flare of any type during the 12-month follow-up period was 76 


\begin{tabular}{|c|c|c|}
\hline Treatment and Patient & Clinical Manifestation & SELENA-SLEDAI Composite Criteria Fulfilled $\grave{\dagger}$ \\
\hline \multicolumn{3}{|l|}{ Oral contraceptives } \\
\hline Patient 1 & $\begin{array}{l}\text { Nephritis and multisystem } \\
\text { flare }\end{array}$ & $\begin{array}{l}\text { Instrument score }>12 \\
\text { New or worsening manifestation } \mathbb{} \\
\text { Increase in dose of prednisone or addition of other drug } \emptyset \\
\text { Increase in physician's global assessment to }>2.5\end{array}$ \\
\hline Patient 2 & Multisystem flare & 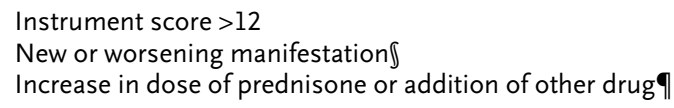 \\
\hline Patient 3 & Multisystem flare\| & $\begin{array}{l}\text { New or worsening manifestation } \mathbb{} \\
\text { Hospitalization for lupus activity }\end{array}$ \\
\hline Patient 4 & Abdominal vasculitis & $\begin{array}{l}\text { New or worsening manifestation } \mathbb{} \\
\text { Increase in dose of prednisone or addition of other drug } \\
\text { Hospitalization for lupus activity }\end{array}$ \\
\hline Patient 5 & Thrombocytopenia & New or worsening manifestation $\mathbb{R}$ \\
\hline Patient 6 & Severe rash & Increase in dose of prednisone or addition of other drug \\
\hline Patient 7 & Fever & 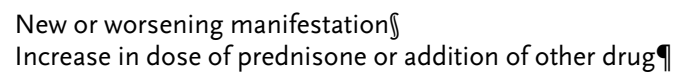 \\
\hline \multicolumn{3}{|l|}{ Placebo } \\
\hline Patient 1 & Nephritis & $\begin{array}{l}\text { Instrument score }>12 \\
\text { New or worsening manifestation } \mathbb{S}\end{array}$ \\
\hline Patient 2 & Nephritis & New or worsening manifestation $\mathbb{S}$ \\
\hline Patient 3 & Nephritis & $\begin{array}{l}\text { New or worsening manifestation } \mathbb{} \\
\text { Hospitalization for lupus activity }\end{array}$ \\
\hline Patient 4 & Nephritis & $\begin{array}{l}\text { New or worsening manifestation } \mathbb{} \\
\text { Increase in physician's global assessment to }>2.5\end{array}$ \\
\hline Patient 5 & Pleural effusions & Hospitalization for lupus activity \\
\hline Patient 6 & CNS involvement & $\begin{array}{l}\text { New or worsening manifestation } \mathbb{} \\
\text { Hospitalization for lupus activity }\end{array}$ \\
\hline Patient 7 & Myositis & $\begin{array}{l}\text { New or worsening manifestation } \mathbb{} \\
\text { Hospitalization for lupus activity }\end{array}$ \\
\hline
\end{tabular}

* SELENA denotes Safety of Estrogen in Lupus Erythematosus National Assessment, SLEDAI Systemic Lupus Erythematosus Disease Activity Index, and CNS central nervous system.

$\uparrow$ Only 3 of 14 severe flares were identified by the SELENA-SLEDAI instrument score, and in each case another criterion was met.

Severe flare occurred after the qualifying visit but before the initiation of study drug.

$\int$ New or worsening manifestations included central nervous system involvement, vasculitis, glomerulonephritis, myositis, thrombocytopenia (platelet count, $<60,000$ per cubic millimeter), or hemolytic anemia (hemoglobin level, $<70 \mathrm{~g}$ per liter, or a drop in hemoglobin of $>30 \mathrm{~g}$ per liter within two weeks), each requiring a doubling of the dose of corticosteroids or an increase to $>0.5 \mathrm{mg}$ per kilogram per day or hospitalization.

I This condition required an increase in the dose of prednisone or its equivalent to more than $0.5 \mathrm{mg}$ per kilogram per day or the initiation of cyclophosphamide, azathioprine, mycophenolate mofetil, or methotrexate.

|| Severe flare occurred after the discontinuation of study drug.

percent for subjects receiving oral contraceptives and 69 percent for subjects receiving placebo, a treatment difference of 0.07 ( $\mathrm{P}=0.34$; upper limit of the one-sided 95 percent confidence interval, 0.19). The estimated relative risk in the oral-contraceptive group by the Cox proportional-hazards model based on the time to the first flare of any type was $1.09(\mathrm{P}=0.65$; 95 percent confidence interval, 0.76 to 1.55$)$.
The mean change in SELENA-SLEDAI scores was not significantly different between groups at any follow-up visit (Table 3). Linear mixed-effects models fitted to these repeated measures found no significant effect due to treatment group $(\mathrm{P}=0.94)$.

\section{ADVERSE EVENTS}

Serious adverse events and adverse events requiring the discontinuation of study medications are 


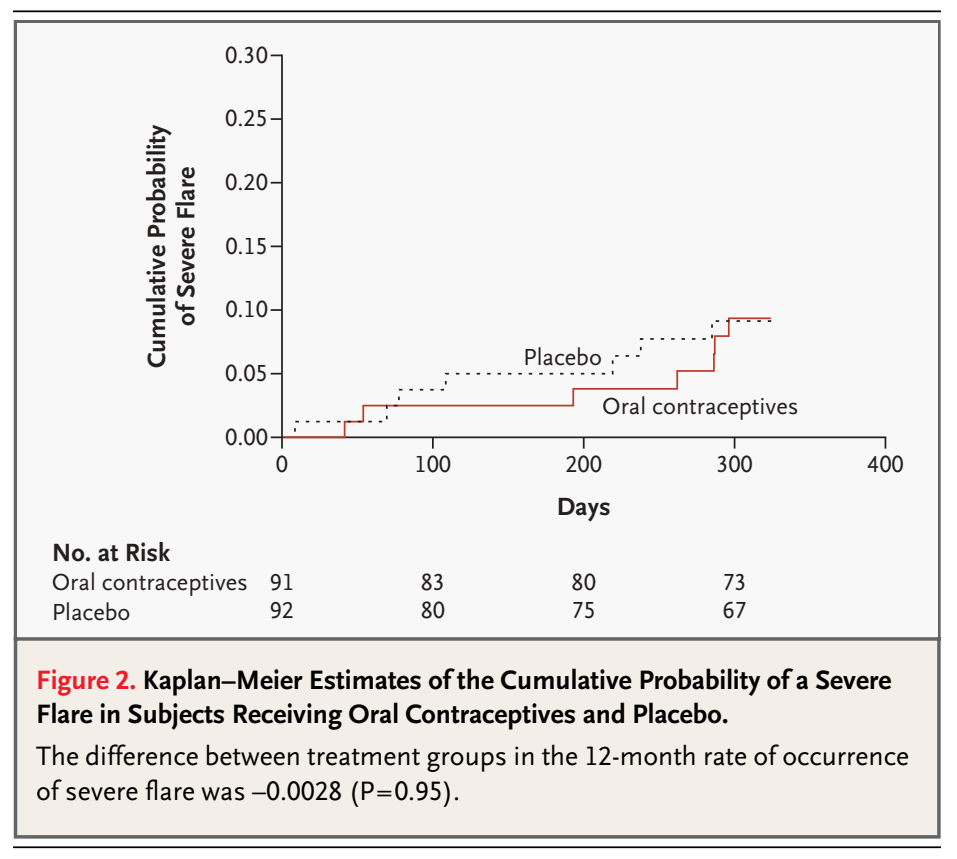

\begin{tabular}{|c|c|c|c|c|c|}
\hline \multirow[t]{2}{*}{ Month } & \multicolumn{2}{|c|}{ Oral Contraceptives } & \multicolumn{2}{|c|}{ Placebo } & \multirow[t]{2}{*}{ P Value $\bar{\dagger}$} \\
\hline & $\begin{array}{l}\text { No. of } \\
\text { Patients }\end{array}$ & Mean Change & $\begin{array}{l}\text { No. of } \\
\text { Patients }\end{array}$ & Mean Change & \\
\hline 1 & 75 & $-0.03 \pm 2.8$ & 79 & $0.06 \pm 2.4$ & 0.83 \\
\hline 2 & 80 & $-0.16 \pm 3.2$ & 74 & $0.03 \pm 2.9$ & 0.70 \\
\hline 3 & 83 & $-0.19 \pm 2.7$ & 79 & $0.22 \pm 2.9$ & 0.35 \\
\hline 6 & 82 & $-0.18 \pm 2.8$ & 75 & $-0.17 \pm 3.0$ & 0.98 \\
\hline 9 & 77 & $0.07 \pm 2.8$ & 70 & $0.06 \pm 2.8$ & 0.99 \\
\hline 12 & 78 & $0.12 \pm 2.9$ & 73 & $-0.11 \pm 2.7$ & 0.62 \\
\hline
\end{tabular}

* Plus-minus values are \pm SD. SELENA denotes Safety of Estrogen in Lupus Erythematosus National Assessment, and SLEDAI Systemic Lupus Erythematosus Disease Activity Index.

$\dagger P$ values are calculated with the use of a two-sided t-test.

\section{DISCUSSION}

Effective and safe birth control is essential to the care of premenopausal women with systemic lupus erythematosus. Hormone-based contraception remains the most effective reversible form of birth control and is the nonsurgical method used by most women (both whites and blacks) between the ages of 15 and 44 years in the United States. ${ }^{13}$ However, available data affirm that women with systemic lupus erythematosus use oral contraceptives less often than do age-matched healthy controls, ${ }^{3}$ reflecting prescribing practices. Yet, the impression that exogenous estrogens may negatively influence lupus disease activity is not derived from any reproducible direct evidence. The SELENA trial prospectively evaluated the effect of oral contraceptives on lupus disease activity. Although the sample was smaller than was initially planned, the trial demonstrated that contraception with the use of estrogen and progesterone does not increase a patient's risk of severe or mild-moderate flare beyond a clinically acceptable margin. The nonadherence rate was consistent with rates among first-time users of oral contraceptives in the general population. ${ }^{13,14}$ In a similar manner, the pregnancy rate of 1 percent in subjects who were receiving oral contraceptives is consistent with national averages. ${ }^{13}$

Current decision making about the use of oral contraceptives in women with systemic lupus erythematosus is based on lupus models in mice and on anecdotal reports and retrospective casecontrol studies in humans. Among 121,645 women in the Nurses' Health Study, past users of oral contraceptives had a small increase in the risk that systemic lupus erythematosus would develop (relative risk, 1.4; 95 percent confidence interval, 0.9 to 2.1), as compared with those who had never used oral contraceptives. ${ }^{15} \mathrm{~A}$ recent case-control study showed a weak association between the risk of lupus and current or past use of oral contraceptives (adjusted odds ratio, 1.3; 95 percent confidence interval, 0.9 to 2.1). ${ }^{16}$ In a retrospective case-control study of subjects with lupus who had a history of nephritis, Jungers et al. ${ }^{4}$ noted flares in 9 of 26 women during the first three months after the initiation of combined oral contraceptives as compared with none of 11 women receiving progestogenonly formulations. In contrast, Julkunen ${ }^{17}$ observed no difference in flare rates among 31 patients with systemic lupus erythematosus who were taking oral contraceptives, as compared with 31 nonusers. 
In a retrospective survey of 55 women who used oral contraceptives after receiving a diagnosis of systemic lupus erythematosus, 7 reported an exacerbation of disease, which was usually confined to the musculoskeletal system (flare rate, 0.45 per 100 patient-months). ${ }^{3}$

The relevance of establishing the safety of oral contraceptives in systemic lupus erythematosus extends beyond birth control. Oral contraceptives may control cyclic lupus activity. ${ }^{18}$ Estrogens prevent bone loss and increase bone mass, ${ }^{19,20}$ suppress bone-resorbing cytokines such as interleukin-121 and interleukin- $6,{ }^{22}$ and exert positive changes in calcium homeostasis. ${ }^{23}$ In a retrospective cohort of 702 women with lupus, fractures occurred in 12.3 percent, a rate nearly five times that in healthy women. ${ }^{24}$ In 376 women with lupus who were observed prospectively, the use of oral contraceptives was associated with a significantly reduced risk of musculoskeletal damage. ${ }^{11}$ The guidelines of the American College of Rheumatology Task Force on Osteoporosis recommend the administration of oral contraceptives to prevent glucocorticoid-induced osteoporosis in premenopausal women with oligomenorrhea or amenorrhea, ${ }^{25}$ although this benefit has not been prospectively evaluated in women with systemic lupus erythematosus.

In sum, although murine models and retrospective human studies suggested that exogenous estrogens might exacerbate lupus disease activity, the results of the OC-SELENA trial do not support this inference. Oral contraceptives were not associated with an increase in severe, mild-moderate, or total flares over the course of one year, as compared with placebo. These data support the use of oral contraceptives containing estrogen as a birth-control choice for patients with inactive or stable, moderate systemic lupus erythematosus who are at low risk for thrombosis.

Supported by a grant (U01 AR42540, to Dr. Buyon) from the National Institute of Arthritis and Musculoskeletal and Skin Diseases and the Office of Research on Women's Health; and by National Institutes of Health (NIH) grants (AR 43727, to Dr. Petri and the Hopkins Lupus Cohort, and M01-RR 00052 and M01-RR 00096, through the General Clinical Research Centers of Johns Hopkins University and the Hospital for Joint Diseases of New York University School of Medicine).

Dr. Diamond reports having received an NIH grant and Dr. Buyon a subgrant (R01 AI062069) entitled "Understanding the SELENA Trial: Estrogen in Lupus."

We are indebted to the members of the study's data and safety monitoring board for their guidance and generous service: Drs. Matthew H. Liang (chairman), John C. Fletcher, John H. Klippel, Helain J. Landy, Larry R. Muenz, and Rosalind Ramsey-Goldman; to

\begin{tabular}{|c|c|c|}
\hline \multirow[t]{2}{*}{ Type of Event } & $\begin{array}{c}\text { Oral } \\
\text { Contraceptives } \\
(\mathrm{N}=91)\end{array}$ & $\begin{array}{l}\text { Placebo } \\
(\mathrm{N}=92)\end{array}$ \\
\hline & \multicolumn{2}{|c|}{ no. of patients (\%) } \\
\hline $\begin{array}{c}\text { Serious adverse events (requiring } \\
\text { hospitalization) }\end{array}$ & $15(16)$ & $13(14)$ \\
\hline Thrombosis & $2(2) \dagger$ & $1(1) ‡$ \\
\hline Gastrointestinal symptoms & $1(1)$ & $3(3)$ \\
\hline Gynecologic symptoms & $1(1)$ & $1(1)$ \\
\hline Infection & $5(5)$ & $4(4)$ \\
\hline Orthopedic surgery & $3(3)$ & $2(2)$ \\
\hline $\begin{array}{l}\text { Toxic encephalopathy secondary } \\
\text { to phenobarbital }\end{array}$ & 0 & $1(1)$ \\
\hline Suicide attempt & $1(1)$ & 0 \\
\hline Elective thymectomy & $1(1)$ & 0 \\
\hline Ectopic pregnancy & $1(1) \int$ & 0 \\
\hline Death & 0 & $1(1) \ddagger$ \\
\hline $\begin{array}{l}\text { Adverse events (requiring withdrawal } \\
\text { of study drug) }\end{array}$ & $7(8)$ & $12(13)$ \\
\hline Thrombosis & 0 & $2(2) \uparrow q$ \\
\hline Pregnancy & $1(1) \dagger \|$ & $2(2) \dagger * *$ \\
\hline Rash & 0 & $2(2) \dagger$ \\
\hline Gynecologic symptoms & $3(3) \dagger$ & $3(3) \dagger$ \\
\hline Migraine & 0 & $3(3) \dagger$ \\
\hline Abnormal liver-function results & $2(2) \dagger$ & 0 \\
\hline Development of hypertension & $1(1) \dagger$ & 0 \\
\hline
\end{tabular}

* This table does not include the serious adverse events that constituted severe lupus flares, which are listed in Table 2.

$\dagger$ Study medications were withdrawn owing to these events.

Subject had discontinued study drug owing to severe flare at three months, was hospitalized for a deep venous thrombosis three months later, and died one year after placebo was discontinued.

$\int$ This pregnancy does not appear in Figure 1 because pregnancy was not the reason study drug was discontinued; ectopic pregnancy developed two months after the subject stopped study drug owing to rash.

If One subject had an ocular thrombosis after randomization and therefore never received study drug; the other subject had a superficial thrombophlebitis at two months, resulting in the discontinuation of study drug.

$\|$ One subject in the oral-contraceptive group had a positive pregnancy test at the 2-month visit, resulting in the discontinuation of study drug; she miscarried twins at 19 weeks' gestation.

** One subject in the placebo group had a positive pregnancy test after randomization and therefore never received study drug; she delivered a healthy infant at term. Another subject in the placebo group had a positive pregnancy test at the 3-month visit, resulting in the discontinuation of placebo; she elected termination at 16 weeks' gestation.

Dr. Mary Louise Skovron for biostatistical expertise and intellectual contribution to the initial design of the study; and to Marcus Vogel and Jeanie Kantrowitz (Hospital for Joint Diseases pharmacists) for their assistance in the establishment and maintenance of the system for blinded allocation and distribution of study medications. 
APPENDIX

The following investigators and study coordinators participated in the OC-SELENA Trial: Johns Hopkins Hospital, Baltimore - C. Robinson; Hospital for Joint Diseases-New York University School of Medicine, New York — P. Louie; Hospital for Special Surgery, New York V. Kaplan; Louisiana State University Health Sciences Center, Shreveport - L. Green and R. Brouillette; Medical College of Wisconsin, Milwaukee - J. Zrnic; Oklahoma Medical Research Foundation, Oklahoma City — J. James, B. Lee, and F. Shelton; Rheumatology Associates of Long Island, Port Jefferson Station, N.Y. — D. Kaell; St. Luke's-Roosevelt Hospital Center, New York - J.T. Merrill, R. Shriky, and A. Shriky; University of California, Los Angeles - A. Rapkin and W. Chen; University of California, San Francisco - M. Fitzpatrick; University of Michigan, Ann Arbor - C.J.M. Van De Ven, G. Christman, and B. Gilson; University of North Carolina at Chapel Hill — W. Meyer and B. Meier; University of Pennsylvania, Philadelphia - K. Barnhart and L. Loh; University of Texas, Houston - N.-H. Chiu.

REFERENCES

1. Masi AT, Kaslow RA. Sex effects in systemic lupus erythematosus: a clue to pathogenesis. Arthritis Rheum 1978;21:480-4.

2. Lahita RG, Bradlow HL, Fishman J, Kunkel HG. Estrogen metabolism in systemic lupus erythematosus: patients and family members. Arthritis Rheum 1982;25: 843-6.

3. Buyon JP, Kalunian KC, Skovron ML, et al. Can women with systemic lupus erythematosus safely use exogenous estrogens? J Clin Rheumatol 1995;1:205-12.

4. Jungers P, Dougados M, Pelissier C, et al. Influence of oral contraceptive therapy on activity of systemic lupus erythematosus. Arthritis Rheum 1982;25:618-23.

5. Roubinian J, Talal N, Siiteri PK, Sakakian JA. Sex hormone modulation of autoimmunity in NZB/NZW mice. Arthritis Rheum 1979;22:1162-9.

6. Grimaldi CM, Cleary J, Dagtas AS Moussai D, Diamond B. Estrogen alters thresholds for B cell apoptosis and activation. J Clin Invest 2002;109:1625-33.

7. Systemic lupus erythematosus in preg nancy. Ann Intern Med 1981;94:667-77.

8. Buyon JP, Petri MA, Kim MY, et al. The effect of combined estrogen and progesterone hormone replacement therapy on disease activity in systemic lupus erythematosus: a randomized trial. Ann Intern Med 2005;142:953-62.

9. Bombardier C, Gladman DD, Urowitz $\mathrm{MB}$, Caron D, Chang CH. Derivation of the SLEDAI: a disease activity index for lupus patients. Arthritis Rheum 1992;35:630-40.
10. Petri M, Buyon J, Kim M. Classification and definition of major flares in SLE clinical trials. Lupus 1999;8:685-91.

11. Petri M. Musculoskeletal complications of systemic lupus erythematosus in the Hopkins Lupus Cohort: an update. Arthritis Care Res 1995;8:137-45.

12. Tan EM, Cohen AS, Fries JF, et al. The 1982 revised criteria for the classification of systemic lupus erythematosus. Arthritis Rheum 1982;25:1271-7.

13. Trussell J, Kost K. Contraceptive failure in the United States: a critical review of the literature. Stud Fam Plann 1987;18:237-83. 14. Rosenberg MJ, Waugh MS. Oral contraceptive discontinuation: a prospective evaluation of frequency and reasons. Am J Obstet Gynecol 1998;179:577-82

15. Sanchez-Guerrero J, Karlson EW, Liang MH, Hunter DJ, Speizer FE, Colditz GA. Pas use of oral contraceptives and the risk of developing systemic lupus erythematosus. Arthritis Rheum 1997;40:804-8.

16. Cooper GS, Dooley MA, Treadwell EL, St Clair EW, Gilkeson GS. Hormonal and reproductive risk factors for developmen of systemic lupus erythematosus: results of population-based, case-control study. Arthritis Rheum 2002;46:1830-9.

17. Julkunen HA. Oral contraceptives in systemic lupus erythematosus: side-effects and influence on the activity of SLE. Scand Rheumatol 1991;20:427-33.

18. Petri M, Robinson C. Oral contraceptives and systemic lupus erythematosus. $\mathrm{Ar}$ thritis Rheum 1997;40:797-803.
19. Kiel DP, Felson DT, Anderson JJ, Wilson PWF, Moskowitz MA. Hip fracture and the use of estrogens in postmenopausal women: the Framingham Study. N Engl J Med 1987;317:1169-74.

20. Effects of hormone therapy on bone mineral density: results from the Postmenopausal Estrogen/Progestin Interventions (PEPI) trial. JAMA 1996;276:1389-96.

21. Pacifici R, Rifas L, McCracken R, et al. Ovarian steroid treatment blocks a postmenopausal increase in blood monocyte interleukin 1 release. Proc Natl Acad Sci U S A 1989;86:2398-402.

22. Jilka RL, Hangoc G, Girasole F, et al. Increased osteoclast development after estrogen loss: mediation by interleukin- 6 . Science 1992;257:88-91.

23. Gallagher JC, Riggs BL, DeLuca HF. Effect of estrogen on calcium absorption and serum vitamin D metabolites in postmenopausal osteoporosis. J Clin Endocrinol Metab 1980;51:1359-64.

24. Ramsey-Goldman R, Dunn JE, Huang $\mathrm{CF}$, et al. Frequency of fractures in women with systemic lupus erythematosus: comparison with United States population data. Arthritis Rheum 1999;42:882-90.

25. American College of Rheumatology Task Force on Osteoporosis Guidelines. Recommendations for the prevention and treatment of glucocorticoid-induced osteoporosis. Arthritis Rheum 1996;39:1791-801.

Copyright @ 2005 Massachusetts Medical Society.

POWERPOINT SLIDES OF JOURNAL FIGURES AND TABLES

At the Journal's Web site, subscribers can automatically create PowerPoint slides of Journal figures and tables. Click on a figure or table in the full-text version of any article at www.nejm.org, and then click on PowerPoint Slide for Teaching. A PowerPoint slide containing the image, with its title and reference citation, can then be downloaded and saved. 